\title{
Rumo a Uma Arquitetura de Referência para Constituintes de Sistemas de Sistemas de Informação
}

\author{
Priscilla Elizabeth Pereira Batista ${ }^{1}$, Valdemar Vicente Graciano Neto ${ }^{1}$ \\ ${ }^{1}$ Instituto de Informática - Universidade Federal de Goiás (UFG) \\ CEP 743690-900 - Goiânia - GO - Brazil \\ \{priscillaelizabeth, valdemarneto\} ainf.ufg.br
}

\begin{abstract}
The aim of this paper is to present the status of an ongoing master's thesis research on the establishment of a reference architecture for systems that could form a constituent a System-of-Information Systems (SoIS), such as constituents of a smart city. Since systems are usually not prepared to be easily integrated to form a SoIS, the main expected contribution of this work is to create a baseline over which software-intensive systems can be derived to originate systems that are prepared to automatically integrate a SoIS with no need of tailoring or further adaptation/evolution.
\end{abstract}

Resumo. O objetivo deste artigo é apresentar o status da pesquisa de dissertação de mestrado em andamento sobre o estabelecimento de uma arquitetura de referência para sistemas que podem se tornar constituintes de um Sistema de Sistemas de Informação (SoIS), como constituintes de uma cidade inteligente. Como os sistemas geralmente não estão preparados para serem facilmente integrados e formar um SoIS, a principal contribuição esperada deste trabalho é criar subsidios para que sistemas intensivos em software possam ser derivados originando sistemas preparados para integrar automaticamente um SoIS sem necessidade de posterior adaptação/evolução.

\section{Introdução}

Cidades inteligentes estão se tornando uma realidade. Diversos projetos de ambientes inteligentes já estão em funcionamento e em um futuro próximo, tais ambientes serão mais comuns. Tais sistemas (cidades inteligentes) são importantes para melhorar a qualidade de vida das pessoas e auxiliar o desenvolvimento sustentável [Mendes et al. 2018].

Cidades Inteligentes são consideradas SoIS (Systems of Information Systems Sistemas de Sistemas de Informação), isto é, uma categoria de SoS (Systems of Systems - Sistemas de Sistemas) onde um ou mais de seus sistemas constituintes são sistemas de informação (SI), podendo ou não haver outros tipos de sistemas incluídos [Graciano Neto et al. 2017]. Vale ressaltar que as siglas SoS e SoIS são usadas tanto para o termo no pural quanto no singular. A heterogeneidade dos constituintes torna suas funcionalidades potencialmente complementares para atingir um objetivo mais amplo. Assim, a interoperabilidade torna-se um fator vital para a entrega de comportamentos mais complexos do SoIS [Fernandes et al. 2018]. Uma vez que o SoIS é uma categoria de SoS, torna-se necessário entender que os SoS têm como características fundamentais, que também se aplicam aos SoIS [Graciano Neto et al. 2018]:

- independência operacional: cada sistema constituinte opera de forma independente em suas atividades internas; 
- independência gerencial: os sistemas constituintes possuem gerentes específicos de forma independente do gerenciamento do SoS;

- distribuição: os sistemas constituintes estão distribuídos;

- desenvolvimento evolutivo: cada sistema constituinte evolui individualmente e causam a evolução do SoS como consequência;

- comportamento emergente: comportamentos complexos são obtidos da combinação dos comportamentos individuais dos constituintes e suas relações;

- arquitetura dinâmica: sistemas constituintes podem entrar e sair do SoS a qualquer momento, modificando a arquitetura em tempo de execução.

Para o desenvolvimento de sistemas complexos como o SoIS é necessário haver uma uniformização no tocante aos constituintes envolvidos para lidar com a variabilidade de suas plataformas, tecnologias e interfaces. É nesse âmbito que entra a AR (Arquitetura de Referência) para constituintes. Este tipo de arquitetura permite a padronização no desenvolvimento de software, facilitando o reaproveitamento de recursos e possibilitando maior interoperabilidade entre os SIs [Nakagawa et al. 2017]. Nesse sentido as ARs se tornam uma poderosa ferramenta para garantir a interoperabilidade dos SIs que irão compor o SoIS, estabelecendo contratos e padrões que permitem a interoperabilidade entre os constituintes.

A Seção 2 traz a apresentação do problema, a 3 apresenta a proposta de solução e métodos de pesquisa. Na Seção 4 tem-se o projeto de avaliação e na 5 as atividades realizadas. As considerações finais são apresentadas na Seção 6.

\section{Apresentação do Problema}

Os SoIS são compostos de sistemas independentes. De acordo com Klein e Vliet [2017] existem diversas barreiras para que os sistemas possam trabalhar colaborativamente. Entre elas podem ser citadas (i) a independência existente no gerenciamento e objetivos de cada sistema, (ii) ausência de um arquiteto do constituinte que possa colaborar com o arquiteto do SoIS e (iii) ainda há a falta de continuidade nos projetos de software, entre outras dificuldades mencionáveis.

Devido a tais dificuldades é considerado de suma importância o desenvolvimento de uma AR para criar padrões no desenvolvimento de sistemas que possuem necessidade de interoperar entre si [Nakagawa e Oliveira 2011]. Além disso, sistemas de maior qualidade podem ser desenvolvidos, promovendo reúso. As ARs também servem de base de conhecimento para os domínios aos quais ela se aplica, podendo ser utilizadas também para pesquisa. Sendo tais arquiteturas específicas de domínio cabe uma análise mais profunda para verificar a possibilidade da criação de uma AR que possa especificar o desenvolvimento de SIs constituintes de um SoIS. Não foram encontrados trabalhos diretamente relacionados à aplicação de AR em constituinte de SoIS. Entretanto Volpato et al. [2017] apresentou uma análise de diversas arquiteturas de referência de sistemas individuais, que não foram planejados para ser parte de um SoS/SoIS, dos mais variados domínios. Dentre elas vale destacar a AUTOSAR (https://www.autosar.org/) do domínio automotivo.

Como atualmente existe uma carência padrões que indiquem como desenvolver um SI garantindo que ele possa se tornar um constituinte de um SoIS, como foi percebido em um mapeamento sistemático realizado no início desta pesquisa, é neste contexto que se propõe o desenvolvimento desta AR. Com esta AR pretende-se facilitar 
o processo de desenvolvimento de um SI constituinte de SoIS e garantir que os sistemas criados com base nesta arquitetura tenham capacidade de integrar um SoIS.

\section{Proposta de Solução e Método de Pesquisa}

O principal objetivo desta pesquisa é exatamente desenvolver uma AR para constituintes de SoIS. Escolheu-se o contexto de cidades inteligentes para restringir o escopo desta pesquisa, uma vez que AR são específicas de domínio. Logo, a AR será elaborada para orientar como um sistema de informação intensivo em software que possa ser constituinte de uma cidade inteligente deveria ser criado.

Para tal desenvolvimento será utilizado o ProSA-RA, que corresponde ao estado da arte em processos para concepção de arquiteturas de referência. Ele é um processo sistematizado criado em etapas para facilitar o desenvolvimento de arquiteturas de referência [Nakagawa et al. 2014]. Ele possui quatro passos como segue:

- Passo 1 - Investigação de Fontes de Informação: feita através de mapeamento sistemático ou revisão sistemática sobre o assunto e tem como principal objetivo ter uma visão geral do que já existe sobre o tema e, principalmente, auxiliar no levantamento de requisitos para o desenvolvimento da AR;

- Passo 2 - Análise Arquitetural: análise das informações obtidas no primeiro passo do processo a fim de identificar as necessidades do sistema e descrever os requisitos para o desenvolvimento da AR;

- Passo 3 - Síntese Arquitetural: descreve-se a AR em si com todas as informações necessárias para que as arquiteturas de software possam se basear;

- Passo 4 - Avaliação Arquitetural: avaliação da AR criada que será feita através de uma lista de verificação baseada no FERA (Framework for Evaluation of Reference Architectures - Estrutura para Avaliação de Arquiteturas de Referência). Trata-se de um questionário com 93 questões de múltipla escolha aplicado às partes interessadas com o intuito de detectar se a AR está apresentada de modo adequado e quais melhorias podem ser aplicadas na AR.

\section{Projeto de Avaliação da Solução}

Uma das etapas do processo de criação da AR é a avaliação da arquitetura. Tal avaliação será composta por um questionário aplicado às partes interessadas conforme citado na Seção 3. A natureza da investigação terá caráter quantitativo e qualitativo. Pretende-se utilizar Grounded Theory para analisar as respostas, além de análise estatística para as respostas a questões objetivas [Strauss e Juliet 1994]. Pretende-se também utilizar a AR para desenvolvimento de um protótipo de SI constituinte de um SoIS.

\section{Atividades Realizadas}

A AR ainda está em fase de desenvolvimento. Dentre as etapas do ProSA-RA citadas na Seção 3, o Passo 1 (Condução de Revisão Sistemática) já está concluído e o 2 (Análise Arquitetural) está em andamento. Tais etapas foram executadas entre os meses de Agosto de 2019 e Fevereiro de 2020. As próximas etapas seguem o planejamento estabelecido para conclusão do projeto de mestrado. 


\section{Considerações Finais}

Este artigo apresentou os resultados preliminares de um projeto de estabelecimento de uma Arquitetura de Referências para Constituintes de SoIS. O intuito do projeto, o método de desenvolvimento, e o plano de avaliação foram apresentados.

Destaca-se como ponto positivo a existência de um processo já consolidado para apoiar o estabelecimento deste tipo de arquitetura. O processo escolhido para este projeto foi o ProSA-RA. Uma etapa do ProSA-RA foi cumprida, ou seja, um mapeamento sistemático foi feito e os requisitos extraídos dele estão sendo analisados como arquiteturalmente significativos para compor a AR.

O próximo passo desta pesquisa é conduzir a terceira etapa do processo com a descrição da AR que será proposta. Posteriormente será dado início à etapa de avaliação da arquitetura. Durante a avaliação é possível que novos requisitos sejam descobertos, isso faz deste um processo iterativo e incremental, onde as etapas podem ser repetidas algumas vezes até chegar a versão final da AR para Constituintes de SoIS. Espera-se que, ao final do projeto, a AR possa auxiliar no aumento da qualidade dos sistemas desenvolvidos para serem parte de um SoIS, em particular reforçando atributos de qualidade dos constituintes (tais como reúso, confiabilidade, e adaptabilidade) e do SoIS como um todo (confiabilidade e adequabilidade funcional).

\section{Referências}

Fernandes, J. C., et al. (2018) "Interoperability in Systems-of-Information Systems: A Systematic Mapping Study", in SBQS. ACM, Curitiba, Brazil, pp. 131-140.

Graciano Neto, V. V., Cavalcante, E., El Hachem, J. e Santos, D. S. (2017) "On the Interplay of Business Process Modeling and Missions in Systems-of-information Systems," in JSOS at ICSE. IEEE, Buenos Aires, pp. 72-73.

Graciano Neto, V. V., et al. (2018) "A Study on Goal Specification for Systems-ofInformation Systems: Design Principles and a Conceptual Model", in SBSI. ACM, Caxias do Sul, RS, p. 21.

Klein, J., e Vliet, H. (2017) "System-of-Systems Viewpoint for System Architecture Documentation", VU University, Amsterdam, Netherlands.

Mendes, A., et al. (2018) "Mandala: an Agent-Based Platform to Support Interoperability in Systems-of-systems," in 6th SESoS. ACM, pp. 21-28.

Nakagawa, E.Y., e Oliveira, L.B.R. (2011) "Using Systematic Review to Elicit Requirements of Reference Architectures", USP, São Carlos, SP.

Nakagawa, E.Y., et al. (2014) "Consolidating a Process for the Design, Representation, and Evaluation of Reference Architectures", IEEE Conference on Software Architecture, Sydney, pp. 143-152.

Nakagawa, E.Y., et al. (2017) "Software Architecture and Reference Architecture of Software-intensive Systems and Systems-of-Systems: Contributions to the State of the Art", in ECSA, ACM, Canterbuty, UK, pp. 4-11.

Strauss, A., e Juliet C. (1994) "Grounded theory methodology." Handbook of qualitative research 17, pp. 273-85.

Volpato, T., et al. (2017) “Two Perspectives on Reference Architecture Sustainability”, in ECSA, ACM, Canterbuty, UK, pp. 188-194. 